Noname manuscript No.

(will be inserted by the editor)

\title{
Photospheric and Subphotospheric Dynamics of Emerging Magnetic Flux
}

\author{
A. G. Kosovichev
}

Received: date / Accepted: date

\begin{abstract}
Magnetic fields emerging from the Sun's interior carry information about physical processes of magnetic field generation and transport in the convection zone. Soon after appearance on the solar surface the magnetic flux gets concentrated in sunspot regions and causes numerous active phenomena on the Sun. This paper discusses some properties of the emerging magnetic flux observed on the solar surface and in the interior. A statistical analysis of variations of the tilt angle of bipolar magnetic regions during the emergence shows that the systematic tilt with respect to the equator (the Joy's law) is most likely established below the surface. However, no evidence of the dependence of the tilt angle on the amount of emerging magnetic flux, predicted by the rising magnetic flux rope theories, is found. Analysis of surface plasma flows in a large emerging active region reveals strong localized upflows and downflows at the initial phase of emergence but finds no evidence for large-scale flows indicating future appearance a large-scale magnetic structure. Local helioseismology provides important tools for mapping perturbations of the wave speed and mass flows below the surface. Initial results from SOHO/MDI and GONG reveal strong diverging flows during the flux emergence, and also localized converging flows around stable sunspots. The wave speed images obtained during the process of formation of a large active region, NOAA 10488, indicate that the magnetic flux gets concentrated in strong field structures just below the surface. Further studies of magnetic flux emergence require systematic helioseismic observations from the ground and space, and realistic MHD simulations of the subsurface dynamics.
\end{abstract}

Keywords Solar magnetism · Magnetic flux · Active regions · Sunspots · Helioseismology

\section{Introduction}

The current paradigm is that the solar magnetic fields are generated by a dynamo action deep in the convection zone, presumably, at the bottom, in a thin rotational shear layer called tachocline. In the tachocline the solar differential rotation changes from the differential rotation of the convection zone to a solid-body rotation of the radiative core. Most of the tachocline is located in a convectively stable zone mixed by convective overshoot

Stanford University, Stanford, CA 94305, USA

E-mail: AKosovichev@ solar.stanford.edu 


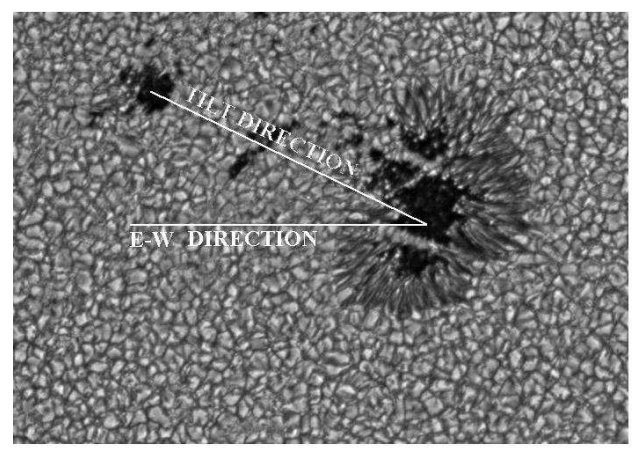

a)

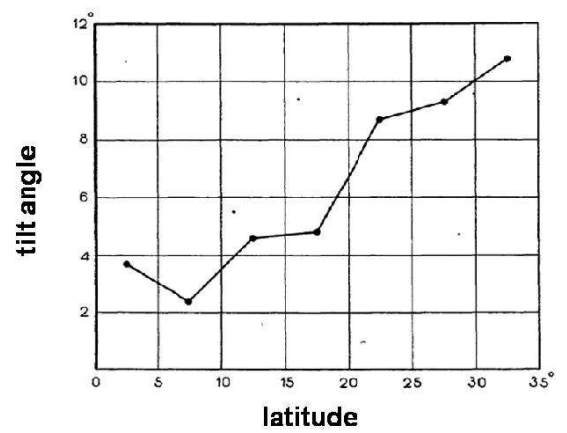

b)

Fig. 1 a) Illustration of the Joy's law (courtesy of D.Hathaway). b) The tilt angle as a function of latitude (Hale et al., 1919).

(Kosovichev, 1996). The combination of strong shearing flows and stability makes possible generation and storage of magnetic field in the tachocline (Parker, 1993).

When the magnetic field is sufficiently strong it becomes buoyant and emerges in the form of toroidal flux ropes (" $\Omega$-loops") oriented in the East-West direction forming bipolar active regions on the surface. The rising magnetic loops are affected by the Coriolis force, which induces retrograde flows, directed from the leading part of the toroidal tube towards its following part. The Coriolis force acting on these flows causes deflection of the flux tubes to higher latitudes and also a tilt with respect to the equator. However, observations show that the magnetic flux emerges mostly at mid and low latitudes, and calculations demonstrate that to explain this the magnetic field generated at the bottom of the convection zone must be very strong, 60-160 kG (D’Silva, 1992; D'Silva and Howard, 1994; Parker, 1994). This is significantly higher than the field strength estimated from energy equipartition with convective motions. Whether this is possible is under debate (e.g. Schüssler, 2005).

In addition, the Coriolis force causes a tilt in the orientation of emerging flux tubes with respect to the equator. This phenomenon is well-known as the Joy's law. Statistical studies by A. H. Joy (Hale et al., 1919) of long series of sunspot drawings showed that the following spot of a bipolar group tend to appear farther from the equator than the preceding spot, and that the higher the latitude the greater the inclination of the axis to the equator Fig. 1 . The tilt of bipolar magnetic groups appears only statistically. The orientation of individual active regions may vary quite significantly. This effect is important for understanding the process of magnetic flux emergence. Also, it is a key element of solar-cycle theories (e.g. Wang and Sheeley, 1991).

Theories of rising magnetic flux tubes seem to explain the tilt (Schmidt, 1968). In these theories, the tilt angle is determined by the torque balance between magnetic tension and the Coriolis forces, and, thus, depends on both the amount of magnetic flux and the emergence latitude. Fisher et al. (1995) and Caligari et al. (1995) found from an analysis of sunspot group data that the theoretically predicted flux dependence of the tilt angle was consistent with the data. Tian and Liu (2003) updated these results using magnetic fluxes instead of polarity separations for a more direct comparison with the theory. Their results showed a less clear agreement between the rising flux tube theories and the observations. These theories also predict that after the rising phase the magnetic flux tubes become stationary, and since the Coriolis force disappears the tilt should decrease under the action of magnetic 
tension in the East-West direction. However, the tilt of bipolar groups does not disappear after the emergence (Howard, 2000). Moreover, there is a tendency of the tilt angle to rotate towards the averaged value defined by the Joy's law. This cannot be explained by the Coriolis effect, and is probably related to a complicated interaction between the magnetic structures and flows below the surface. An alternative explanation suggested by Babcock (1961) is that the tilt is due to the spiral orientation of the magnetic field lines below the surface, wrapped around the Sun by the differential rotation. However, his mechanism assumes that the toroidal field is generated close to the surface, not in the tachocline.

In general, understanding of the observed properties of emerging magnetic flux is closely related to the depth of the main dynamo process in the Sun. While the modern theories assume that the solar dynamo operates in the tachocline there is no convincing observational evidence to support this. Also, there are theoretical difficulties. The pros and cons of the dynamo mechanisms operating in the tachocline and in the bulk of the convection zone, or perhaps even in the near-surface shear layer, are discussed by Brandenburg (2005). From the observational point of view, helioseismology observations for the whole solar cycle from the Michelson Doppler Imager (MDI) instrument on Solar and Heliospheric Observatory (SOHO) (Scherrer et al., 1995) and Global Oscillation Network Group (GONG) (Harvey et al., 1996) do not provide a convincing evidence for solar-cycle variations of the solar rotation rate in the tachocline (Howe et al., 2007). Such variations are expected because of the back reaction of the strong dynamo-generated magnetic fields on the turbulent Reynolds stresses and, hence, on the differential rotation (which is maintained by the Reynolds stresses). Moreover, the comparison of the rotation rate of long-living complexes of activity (which are the sources of repeated flux emergence) with the internal differential rotation deduced from helioseismology showed that the roots of the complexes of activity are probably located in the near-surface shear layer (Benevolenskaya et al., 1999). Determination of the depth of the solar dynamo is probably one of the most important problems of solar magnetism.

Observations show that emerging magnetic flux plays an important role in initiation of solar flares and coronal mass ejections. Thus, it is important to develop predicting capabilities for flux emergence. This problem can be addressed by helioseismology but initial attempts to detect the magnetic flux in the interior before it becomes visible on the surface showed that this is difficult because of the high emergence speed in the upper $20 \mathrm{Mm}$ (Kosovichev et al., 2000). Thus, it is important to investigate large-scale flow patterns, which may provide indication of the flux emergence and development of large magnetic regions in the interior.

In general, investigation of emerging magnetic flux includes the following questions:

- How deep is the source of emerging magnetic flux?

- Does emerging magnetic flux become disconnected from the source?

- Why does magnetic flux tend to emerge in the same areas, forming complexes of activity?

- What is the plasma dynamics associated with emerging flux?

- How does emerging flux interact with the existing magnetic fields, and triggers flares and CMEs?

- Can we predict emerging magnetic flux before it become visible on the surface?

- Can we predict evolution of active regions?

This paper discusses some of these questions and presents recent results of investigation of surface and subsurface characteristics of the magnetic flux emergence process obtained from SOHO/MDI and GONG. In particular, I discuss a new analysis of the Joy's law for the 
emerging flux, dynamics of the photospheric plasma prior and during the flux emergence, methods and results of acoustic tomography of wave-speed perturbations and mass flows below the visible surface, and also future observational projects and perspectives.

\section{Observations of Emerging Magnetic Flux in the Photosphere}

\subsection{Joy's Law and Magnetic Flux Transport}

The tilt of bipolar sunspot groups with respect to the equator (the Joy's law) is one of the fundamental properties of solar magnetism. This phenomenon is closely related to the dynamo mechanism and the process of flux emergence. The key question is whether the tilt is caused by the Coriolis force acting on magnetic flux tubes radially moving from the bottom of the convection zone (Schmidt, 1968), or it reflects the orientation of subsurface magnetic field lines stretched by the differential rotation (Babcock, 1961), or it is created by subphotospheric shearing flows after the emergence (Howard, 1996). Previous studies of the Joy's law were based on daily white light images or magnetograms of sunspot group. These data did not have sufficient temporal resolution to investigate variations of the tilt angle during the flux emergence process.

Using a series of 96-min cadence magnetograms from SOHO/MDI, Kosovichev and Stenflo (2008) attempted to investigate the tilt angle and its statistical relationships to the region latitude, the amount of emerging flux, the emergence rate and the separation between the magnetic polarities. The magnetograms obtained almost uninterruptedly for almost the whole solar cycle, from May 1996 until October 2006, have been analyzed. During this period the MDI instrument on SOHO observed more than 2000 active regions, and 715 active regions, which emerged within 30 degrees from the central meridian, are selected for this study.

The analysis method is pretty straightforward. Each active region is remapped into the heliographic coordinates. The tilt angle and the separation between the magnetic polarities are calculated for their centers of gravity. The period of the growth of the total magnetic flux (lasting usually 2-3 days) is divided into 5 intervals, and the statistical relations are calculated for each interval separately, and for the whole emergence phase.

A typical example of this data analysis is shown in Fig. 2 for active region NOAA 8167 emerged in the Southern hemisphere at about $26^{\circ}$ latitude. The magnetic flux of both polarities rapidly and simultaneously increased and reached a maximum during the first 2 days after the initial appearance of the bipolar region on the surface (Fig. 2k). The tilt angle (Fig. 2d) shows rapid variations at the beginning of emergence, reaches a maximum of about $15^{\circ}$ and then stabilizes at about $7-8^{\circ}$. The separation between the polarities (Fig. 22 ) increases during the emergence phase and continues after the total flux reaches the maximum. It starts decreasing as the active region decays. Figures $2 \mathrm{f}-\mathrm{h}$ show the relationships among these properties. In this example the tilt angle is established rather quickly during the emergence in accordance with the Joy's law (the blue polarity is closer to the equator than the red polarity in Fig. 2 $\mathrm{b}$ ). However, the title angle varies significantly in our sample of emerging active regions, and the Joy's law holds only statistically.

Figures $3 \mathrm{a}-\mathrm{c}$ show the distributions of the tilt angles with latitude for three periods of emergence (the total flux growth): the initial appearance (emergence interval 1), the mid interval (3) and at the end of emergence (interval 5). It appears that at the beginning of emergence the tilt angle is randomly distributed, and the mean tilt angle is about zero (Fig. 3 3 ). However, at the middle of the emergence period the distribution of the tilt angle clearly follows the Joy's law (Fig. 3b) with the latitudinal dependence and the mean tilt angle of 


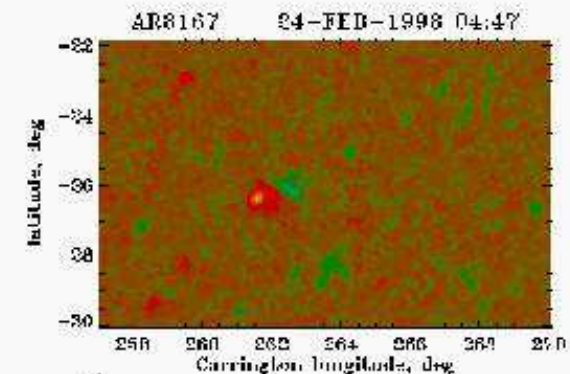

a)

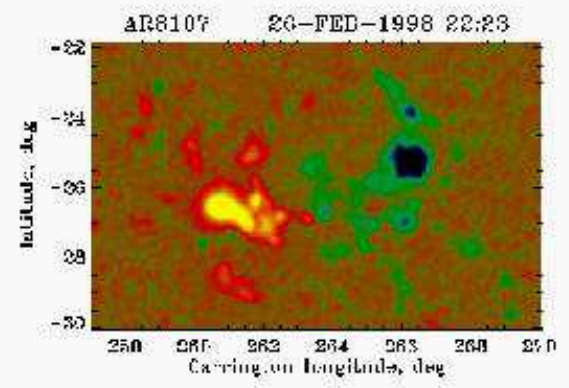

b)

AR8167: 23-FEB-1998_23:59 - 2-MAR-1998_23:59, lat $=-27.0000$, CR lon $=262.000$
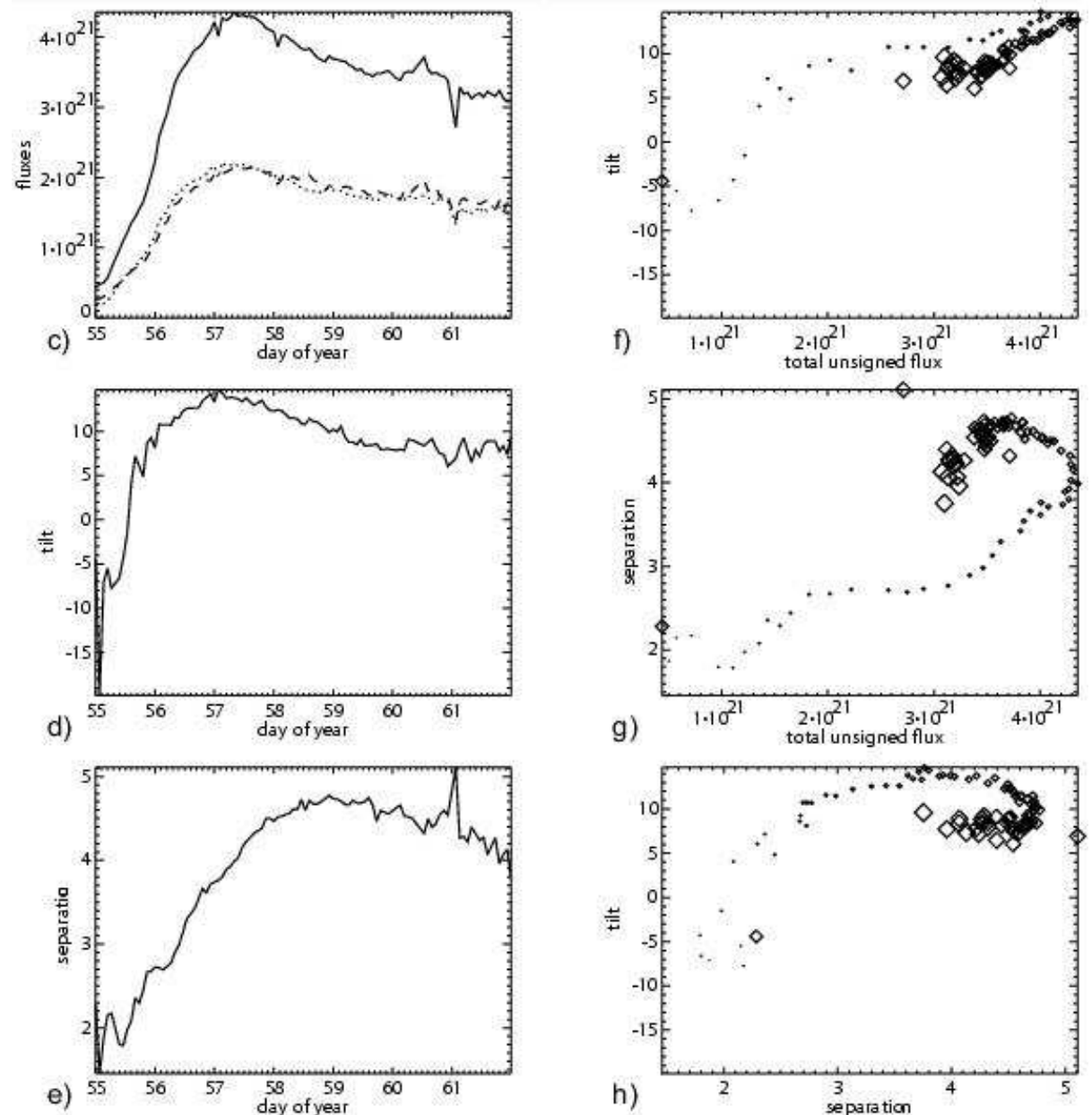

Fig. 2 Magnetograms of active region NOAA 8167: a) at the beginning of flux emergence and b) at the end of emergence. The green and blue colors show the negative polarity; the red and yellow colors show the positive polarity. The evolutions of: c) magnetic fluxes in Mx (dashed curve - positive polarity, dotted curve - negative polarity, the solid curve - the total unsigned flux); d) the tilt angle (in degrees); e) the separation between the polarities (in heliographic degrees). The relationships between: $\mathrm{f}$ ) the tilt angle and total unsigned flux, $g$ ) the polarity separation and the total flux, h) the tilt angle and the separation. The symbol size is proportional to time from the start of emergence. 

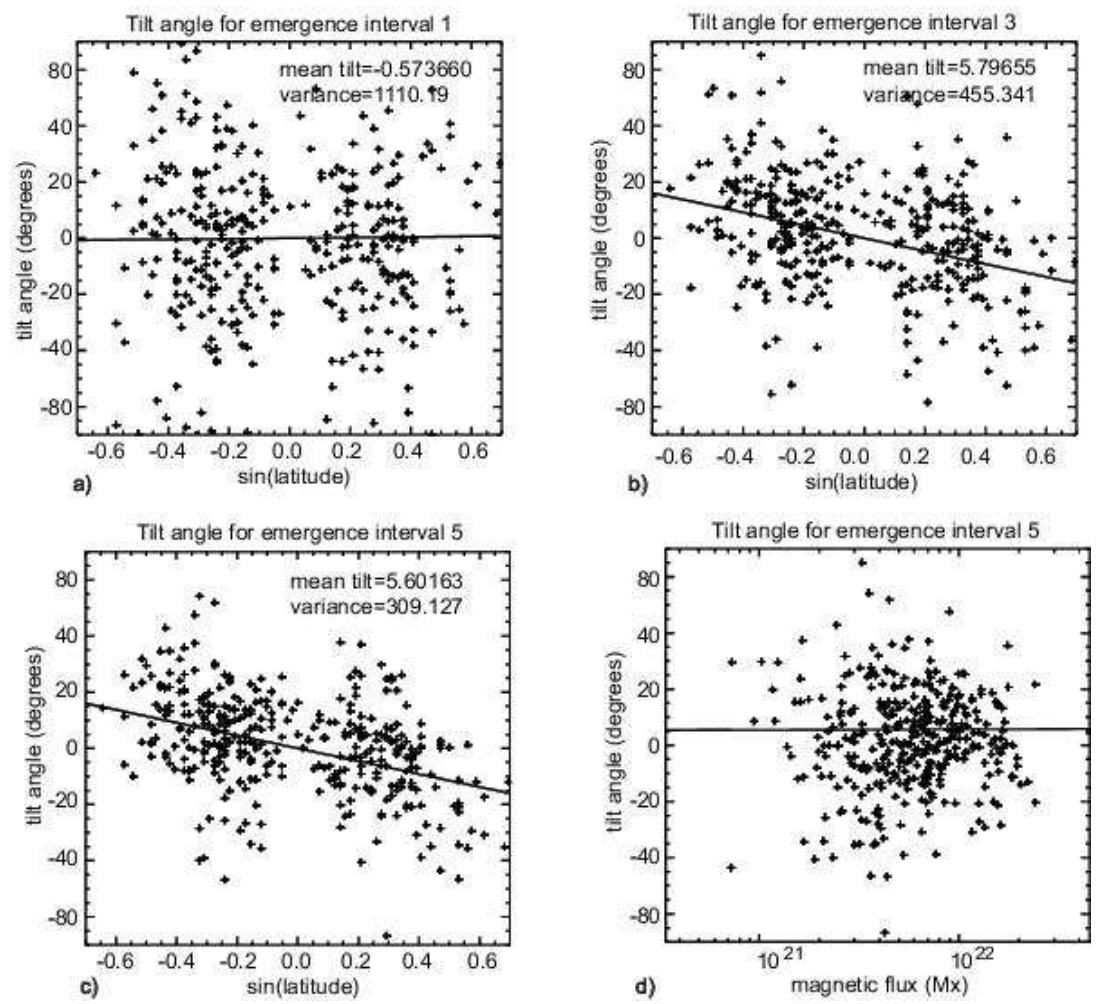

Fig. 3 The distribution of the tilt angle with sine latitude at the beginning of emergence (a), at the middle of the emergence interval (b), and at the end of emergence (c); the distribution of the tilt angle with the total magnetic flux at the end of emergence.

about 6 degrees. At the end of the emergence period the Joy's law distribution becomes more pronounced as the variance of the deviation from the linear dependence on the sine latitude decreases (Fig. 3r). However, these data show no significant correlation between the tilt angle and the total magnetic flux at the end of emergence (Fig. 3 $\mathrm{d}$ ).

These results show that the tilt of bipolar magnetic regions becomes statistically significant during the emergence process. This means that the tilt is established in subsurface layers. Among the mechanisms suggested to explain the observed tilt are: the spiral orientation of the subsurface toroidal field lines wrapped around the differential rotation (Babcock, 1961), the effect of the Coriolis force acting on the flux tubes moving from the bottom of the convection zone (Schmidt, 1968), and large-scale subsurface motions associated with the differential rotation and meridional circulation (Howard, 1996). The most popular explanation that the tilt is caused by the Coriolis force acting on the flows inside an emerging flux rope was questioned by Howard (1996) who investigated variations of the tilt after the emergence and found that the tilt angle moves towards the Joy's law orientation instead of relaxing to the East-West direction as expected from this theory when the radial flux rope motion stops (and, thus, the Coriolis force vanishes). In addition, our results do not show a significant dependence of the tilt on the magnetic flux, predicted by the Coriolis force theories (e.g. Fan et al., 1994). Howard (1996) suggested that the tilt angle may be estab- 

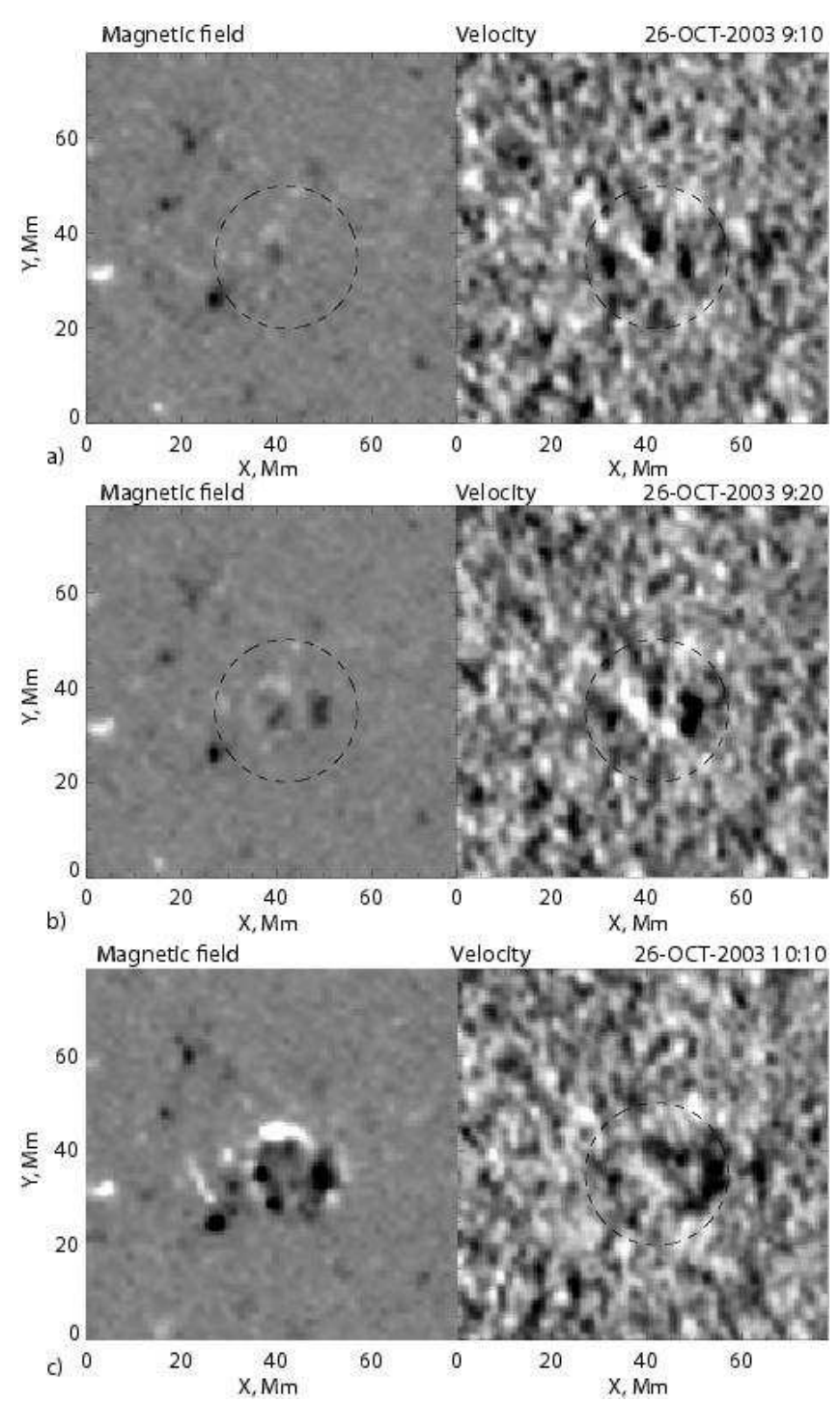

Fig. 4 Maps of the line-of-sight magnetic field (left panels) and Doppler velocity (right panels) on the solar surface obtained from SOHO/MDI at the beginning of emergence of AR 10488, October 26, 2008, a) 7:30 UT, b) 7:40 UT, c) 7:50 UT. The range of the magnetic field strength is $[-180 \mathrm{G}, 180 \mathrm{G}]$. The range of the Doppler velocity is $[-600 \mathrm{~m} / \mathrm{s}, 600 \mathrm{~m} / \mathrm{s}]$. The dark color shows upflows, and the white color shows downflows. The dashed circle outlines the area of the initial magnetic flux emergence.

lished after the emergence due to the action of the depth dependent differential rotation and meridional flow. However, our results indicate that the bipolar magnetic flux regions emerge already tilted in accordance with the Joy's law. The helioseismology results show that the emerging magnetic structures propagate very fast in the upper convection zone (Sec. 3.2), and thus do not support the Howard's idea. Perhaps, we should go back to the Babcock's mechanism that the tilt is caused by the spiral structure of the subsurface toroidal flux tubes. 


\subsection{Mass Flows}

One can expect that when a large magnetic flux rope emerges on the solar surface it drives significant upflows and outflows, which may be detectable when the flux rope is still below the surface. Figure 4 shows the magnetograms and Dopplergrams obtained from SOHO/MDI on October 26, 2003, during the initial emergence of AR 10488, which later grew in to one of the largest active regions of Solar Cycle 23. These data reveal strong localized upflows (dark features in the velocity images) in the places of the initial magnetic flux growth at 9:10 UT (Fig. 4 4 ). Ten min later the upflow velocity reaches a peak of about $800 \mathrm{~m} / \mathrm{s}$, and the magnetic flux starts appearing on the surface (Fig. $4 \mathrm{~b}$ ). The strong upflow is mostly concentrated in the leading part of the emerging flux. In the following part, the data show the Doppler shift of the opposite sign corresponding to downflows. After the appearance of the magnetic flux and its initial growth we observe the similar flow pattern with upflows in the leading part, but the velocity amplitude decreases (Fig.44).

The evolution of the mean Doppler velocity and the total magnetic flux in the area of emergence (Fig. 5) shows a sharp rise of upflows, which continue to be strong for about 2 hours during the initial emergence. The mean velocity reaches $\sim 150 \mathrm{~m} / \mathrm{s}$, well above the mean velocity fluctuations of about $50 \mathrm{~m} / \mathrm{s}$ in similar-sized quiet-Sun regions. After the initial emergence phase, we do not observe significant upflows despite the continuing growth of the active regions.

The strong photospheric plasma flows associated with the magnetic flux emergence of this active region have been also detected by Grigor'ev et al. (2007). It is unclear if such surface flows are typical for emerging magnetic flux and if their strength corresponds to the amount of emerging flux. Pevtsov and Lamb (2006) studied plasma flows in fifteen emerging active regions using Dopplergrams, magnetograms, and white light observations from SOHO/MDI. They observed no consistent plasma flows at the future location of an active region before its emergence.

Also, in the case of AR10488 there is a systematic upflow in the leading magnetic polarity and a downflow in the following polarity. This can interpreted as flows inside the emerging flux tubes, driven by the Coriolis force (e.g. Fan et al., 1994). However, Pevtsov and Lamb (2006) found the asymmetric flows only in three active regions. In two regions, flows are directed from the following to leading polarity, and in one region material flows from the leading to the following polarity. Thus, more detailed statistical studies of the flow dynamics in emerging magnetic flux are necessary.

\section{Investigations of Emerging Flux by Helioseismology}

Methods of local helioseismology developed in recent years allow us to probe physical conditions of the solar plasma below the surface and detect the wave-speed structures and mass flows associated with the emerging magnetic flux.

\subsection{Method of Time-Distance Helioseismology}

Time-distance helioseismology measures travel times of acoustic waves propagating to different distances, and uses these measurements to infer variations of the wave speed along the wave paths. Turbulent convection excites acoustic waves which propagate deep into the solar interior. Because the sound speed increases with depth these waves are refracted and 


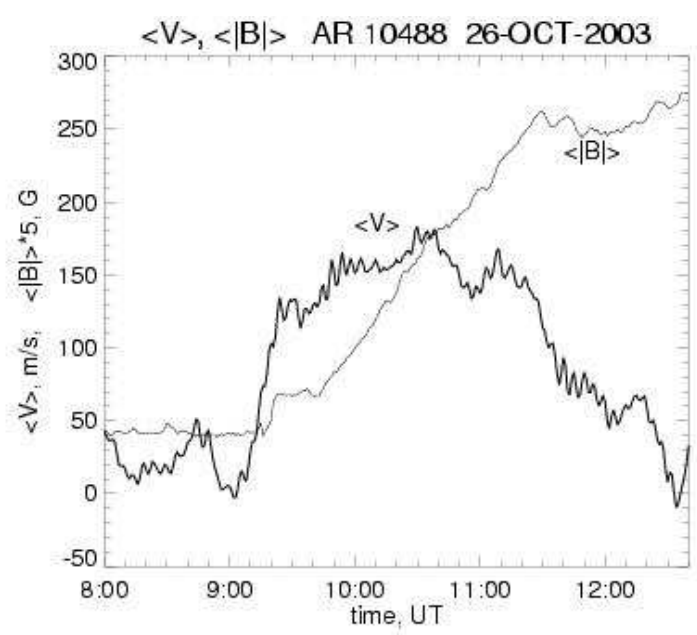

Fig. 5 The mean Doppler velocity and the total magnetic flux as a function of time in the region of the initial emergence of AR 10488. The positive velocity values correspond to the plasma motions towards the observer (upflow).
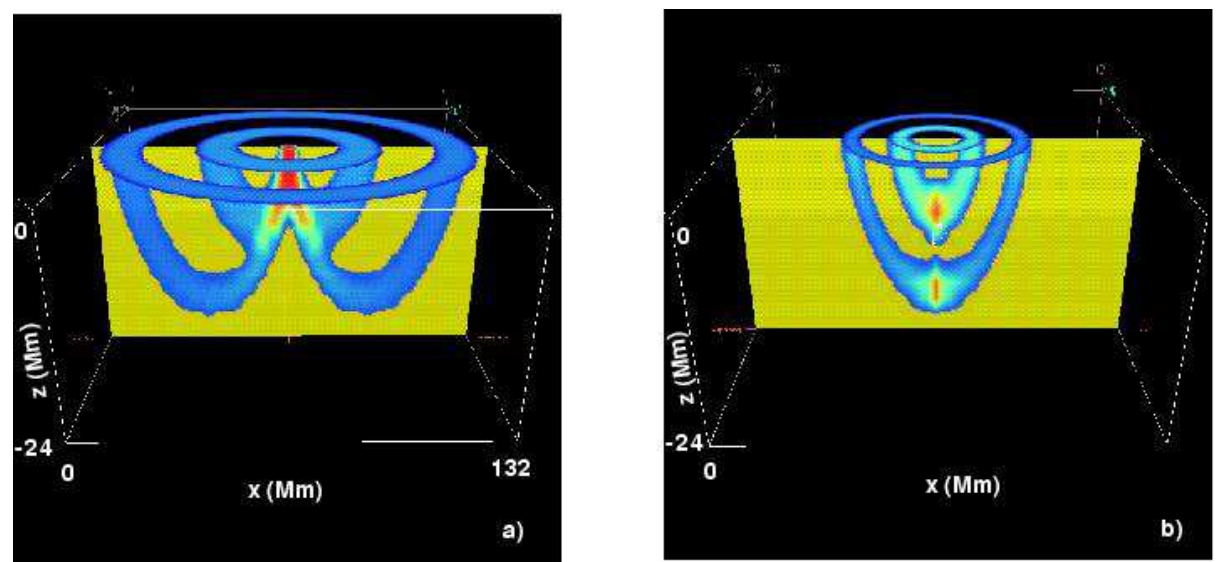

Fig. 6 Illustration of time-distance sensitivity kernels calculated in the ray-path approximation: a) surfacefocusing scheme; b) deep-focusing scheme.

come back to the solar surface. The wave speed depends on the temperature, magnetic field strength and flow velocity field in the region of the wave propagation. By measuring reciprocal travel times of acoustic waves propagating along the same ray paths in opposite directions, and then taking the mean and the difference of these travel times, it is possible to separate the flow velocity (advection) effect from temperature and magnetic field perturbations (Kosovichev and Duvall, 1997). However, in order to disentangle the contributions of temperature variations and magnetic field to the mean travel times it is necessary to measure the travel-time anisotropy, and this has not been accomplished. Therefore, the current 


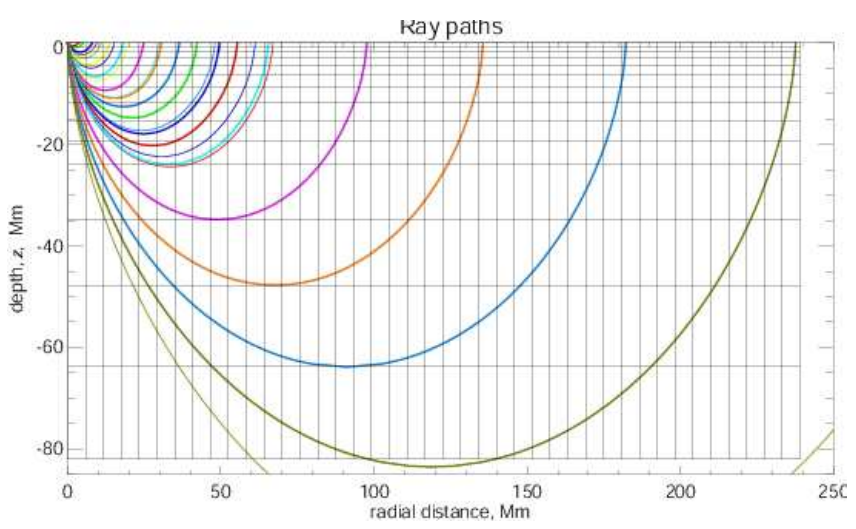

Fig. 7 A vertical cut through the 3D data inversion grid and a sample of acoustic ray paths.

helioseismic results represent maps of sub-photospheric variations of the magneto-acoustic wave speed and flow.

The travel times are typically measured from a cross-covariance function of solar oscillation signals for various distances and time lags. When for a given distance the time lag corresponds to the propagation time of acoustic waves for this distance, a wavepacket-like signal appears in the cross-covariance function. The cross-covariance plotted as a function of the distance and the time lag displays a set of ridges formed by the wave-packet signals, representing an analog of a solar "seismogram". Since the solar oscillations are stochastic it is necessary to use the oscillation signals at least $2-8$ hours long and also average them over some surface (typically, circular) areas in order to obtain a sufficient signal-tonoise ratio. Then, the travel times are determined by fitting a wavelet to this function (e.g. Kosovichev and Duvall, 1997), or by measuring displacement of the ridges (Gizon and Birch, 2002). Two general observing schemes, so-called 'surface-focusing' and 'deep-focusing', have been used in the travel-time measurements. In the surface-focusing scheme the travel times are measured for acoustic waves traveling between a central point and surrounding annuli. In the deep-focusing scheme, the travel times are measured for the acoustic wave packets traveling between the opposite parts of the annuli, the ray paths of which cross each other in a point located below the surface.

The relationship between the observed travel-time variations and the internal properties of the Sun is given by so-called sensitivity kernels (illustrated in Fig. 6 for both surface- and deep focusing) through integral equations. These integral equations are solved by standard mathematical inversion techniques such as Least Square QR Decomposition (LSQR) and Multi-Channel Deconvolution (MCD) (Kosovichev, 1996; Jensen et al., 2001; Couvidat et al., 2006). The sensitivity functions are calculated using a ray theory or more complicated wave perturbation theories, e.g., the Born approximation, which takes into account the finite wave-length effects. These theories can also take into account stochastic properties of acoustic sources distributed over the solar surface (Gizon and Birch, 2002; Birch et al., 2004).

The vertical structure of the computational grid and a sample of acoustic ray paths, used in this paper, are illustrated in Figure 7 The travel times are measured for waves traveling between a central location and surrounding annuli with different radial distances from the central point. The width of the annuli is larger for larger distances in order to improve the 
signal-to-noise ratio. A set of 17 annuli covering the distance range from 0.54 to 24.06 heliographic degrees (from 6.5 to $292 \mathrm{Mm}$ ) was used. The acoustic waves traveling to these distances sample the Sun's interior up to the depth of $95 \mathrm{Mm}$. The central locations of the time-distance measurements are chosen on a uniform $256 \times 256$ grid with the grid step of $2.9 \mathrm{Mm}$. A total of $1.1 \times 10^{6}$ travel time measurements are made to obtain each wave-speed image of the interior. For the flow velocity, the number of the measurements is three times larger, because in this case in addition to the travel times for a whole annulus it is necessary to measure also the travel times for waves traveling North-South and East-West. This is done by dividing each annulus into four sectors. A part of the inversion grid and the ray paths of the acoustic waves are illustrated in Fig. 7). The horizontal step in this figure is twice as large as the step of the travel-time measurements. The vertical grid, $82 \mathrm{Mm}$ deep, is non-uniform with the step size increasing with depth, from $0.7 \mathrm{Mm}$ at the top to $17 \mathrm{Mm}$ at the bottom. The inversion procedure for all three components of flow velocity is described by Kosovichev and Duvall (1997).

\subsection{Tomographic Imaging of Wave-Speed Perturbations}

Figure 8 shows the results for the emerging active region, NOAA 8131, of January, 1998, obtained by Kosovichev et al. (2000). This was a high-latitude region of the new solar cycle which began in 1997. The distribution of the wave speed variations in a vertical cross-section in the region of the emerging flux and in a horizontal plane at a depth of $18 \mathrm{Mm}$ are shown for six 8-hour consecutive intervals. The perturbations of the magnetosonic speed shown in this figure are associated with the magnetic field and temperature variations in the emerging magnetic ropes and in the surrounding plasma. The panel (a) shows no significant variations in the region of emergence, which is at the middle of the vertical plane. The MDI magnetogram shown at the top indicates only very weak magnetic field above this region. The panel (b) shows a slight positive perturbation associated with the emerging region. During the next 8 hours (panel c) the perturbation becomes stronger and occupies the whole range of depths and continue to increase. These results show that the emerging flux propagates very quickly through the upper $18 \mathrm{Mm}$ of the convection zone. We have also analyzed the data for 2-hour intervals at the start of emergence from 2:00 UT to 4:00 UT, January 12, 1998, (Fig. 9) and concluded that the emerging flux propagated through the characteristic depth of $10 \mathrm{Mm}$ in approximately 2 hours. This gives an estimate of the speed of emergence $\approx 1.3$ $\mathrm{km} / \mathrm{s}$. This speed is similar to the speed predicted by the theories of emerging flux ropes. The typical amplitude of the wave-speed variation in the emerging active region is about 0.5 $\mathrm{km} / \mathrm{s}$. After the emergence we observed a gradual increase of the perturbation in the subsurface layers, and the formation of sunspots (Fig. 7 $\mathrm{d}-\mathrm{f}$ ). The observed development of the active region seems to suggest that the sunspots are formed as a result of the concentration of magnetic flux close to the surface.

Several large active regions emerged on the Sun in October-November 2003. This period represents one of the most significant impulses of solar activity in solar cycle 23. During this period the MDI instrument on SOHO was in the full-disk mode ("Dynamics program"), taking full-disk Dopplergrams and line-of-sight magnetograms every minute. Thus, it was able to capture the emergence of active regions NOAA 10488, and also obtain data for two other large active regions, 10484 and 10486. The active region, 10488, emerged in the Northern hemisphere (at $291 \mathrm{deg}$ Carrington longitude and 8 deg latitude) approximately at the same longitude as AR 10486, which emerged earlier and had a very complex magnetic 

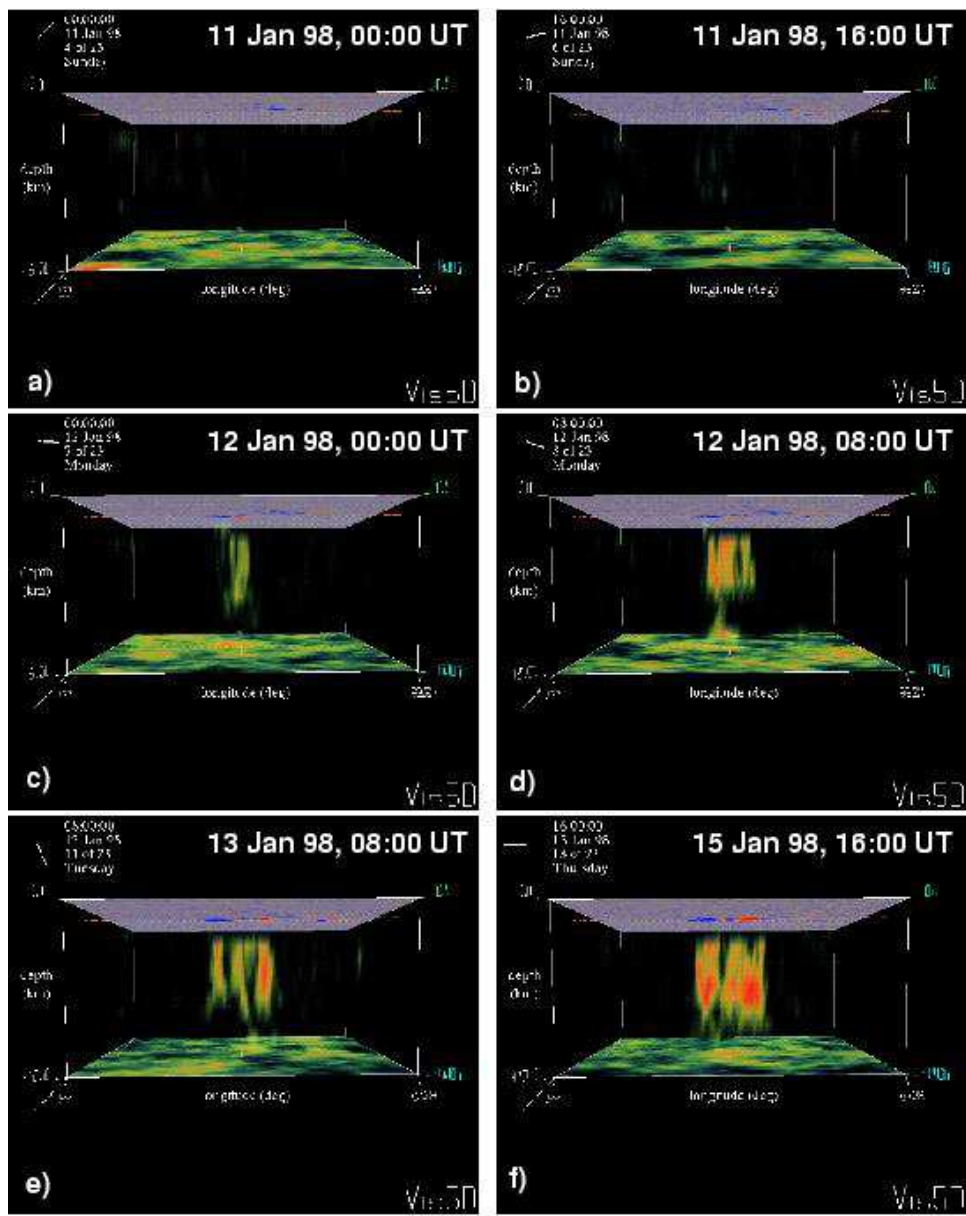

Fig. 8 The wave-speed perturbation in the emerging active region, NOAA 8131. The horizontal size of the box is approximately 38 degrees $(460 \mathrm{Mm})$, the vertical size is $18 \mathrm{Mm}$. The panels on the top are MDI magnetograms showing the surface magnetic field of positive (red) and negative (blue) polarities. The perturbations of the wave speed are approximately in the range from -1 to $+1 \mathrm{~km} / \mathrm{s}$. The positive variations are shown in red, and the negative ones in blue.

configuration resulting in several strong flares. It is possible that these two active regions had a common nest in the interior (Zhou et al., 2007).

Using the method of time-distance helioseismology, we have obtained wave-speed and flow velocity maps for 8 days, 25-31 October, 2003, (Kosovichev and Duvall, 2008). The maps are obtained using 8-hour time series with a 2-hour shift. Total 96 wave-speed and flow maps were obtained. Fig. 10 shows a sample of the wave-speed images. The vertical cut through these images (except the image of 31 Oct 2003, 12:00) is made through both AR 10486 and 10488 in approximately the North-South direction, and for the image of 31 Oct 2003, 12:00, (Fig. 10f) it is made in the East-West direction. The depth of the image box is $48 \mathrm{Mm}$, and the horizontal size is about $540 \mathrm{Mm}$. 


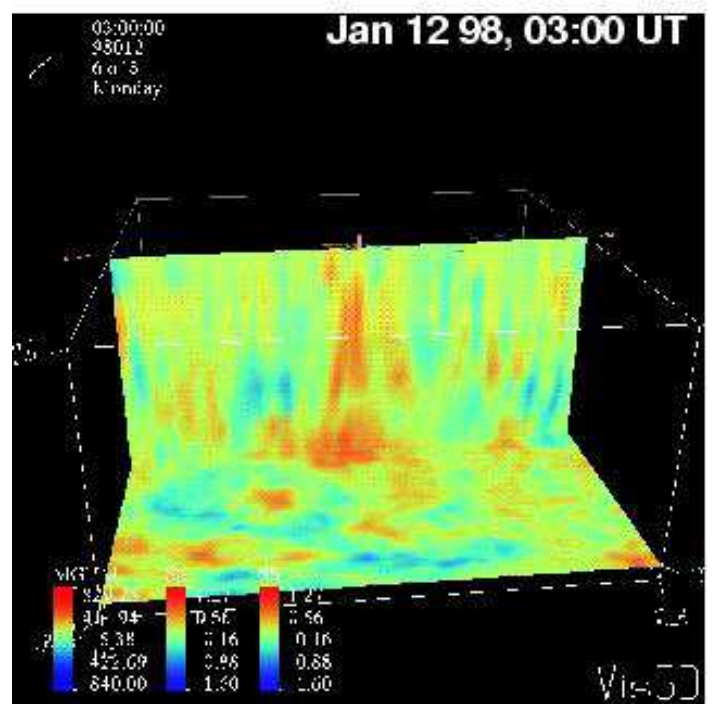

Fig. 9 Image of the magneto-acoustic wave speed in an emerging active region (AR 8131) in the solar convection zone obtained from the SOHO Michelson Doppler Imager (MDI) data on January 12, 1998, from 02:00 to 04:00 UT, using time-distance helioseismology. The horizontal size of the box is approximately 560 $\mathrm{Mm}$, and the depth is $18 \mathrm{Mm}$. The (mostly) transparent panel on the top is an MDI magnetogram showing the surface magnetic field of positive (red) and negative (blue) polarities stronger than 200 Gauss. The vertical and bottom panels show perturbations of the wave speed which are approximately in the range from -1.3 to $+1.3 \mathrm{~km} / \mathrm{s}$. The positive variations are shown in red, and the negative ones in blue. A large active region formed at this location within a day after these observations.

The results show that the first wave-speed signal below the surface appeared in the image obtained on 26 October, 2003, for the time interval centered at 12:00 UT (Fig. 10 p). This is slightly ahead of the growth of the total magnetic field flux, which started to grow at about 20:00 UT (Fig. 111 left, solid curve; however, the first magnetic field signal appeared approximately at the same time). During the next 8 hours, between 12:00 and 20:00, the wave-speed perturbation rapidly grows, and is most visible in the subsurface layers, about $10 \mathrm{Mm}$ deep. In the deeper interior, we do not detect a clear signal above the noise level at this time. This may be because the relative perturbation in these layers is too weak, and also may indicate that the formation of magnetic flux concentrations starts in the subsurface layers. During the next 8 hours the signal extends into the deeper layers and continues to grow (Fig. 10d). The typical two-layer structure with lower wave speed in the top 4-5 Mm, and higher wave speed in the deeper layers is formed (Kosovichev et al., 2000; Jensen et al., 2001; Couvidat et al., 2006). During the following 5 days of the MDI observations, the wave-speed perturbation below the active region becomes larger and stronger, and in the East-West direction it forms a loop-like structure (Fig. 10f). This structure can be traced to the depth of about $30 \mathrm{Mm}$, and then it is lost in noise.

In Figure 11 we compare the evolution of the total (unsigned) magnetic flux of the active regions and the mean wave-speed signal in the two depth intervals, 1-6 Mm and 8-20 Mm. In both cases, the signals correspond well to the evolution of the surface magnetic flux. There is possibly an indication of a slight lead of the wave-speed signal at 1-6 Mm, but there is no 


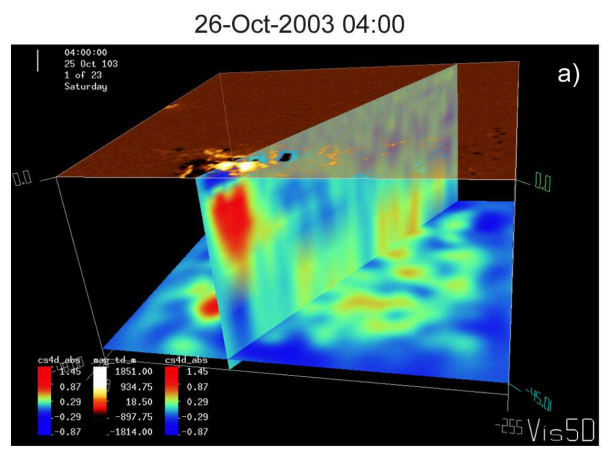

26-Oct-2003 12:00

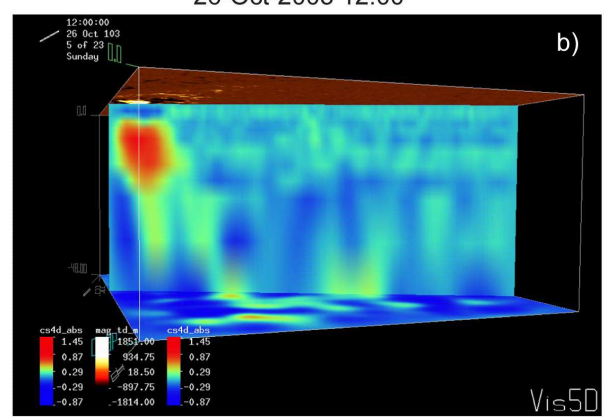

26-Oct-2003 20:00

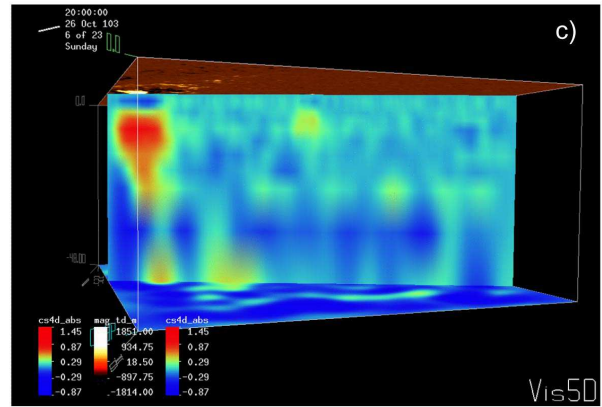

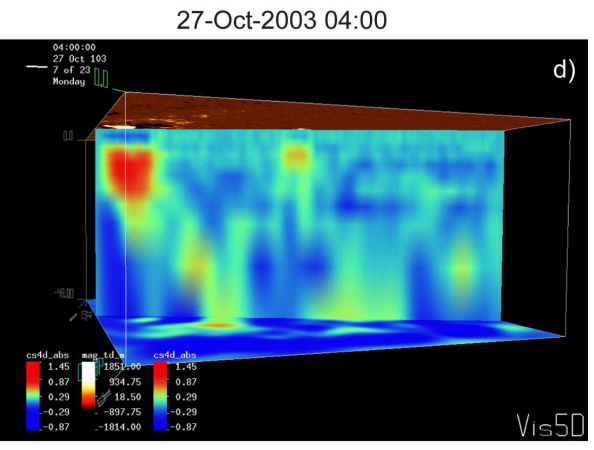

29-Oct-2003 04:00

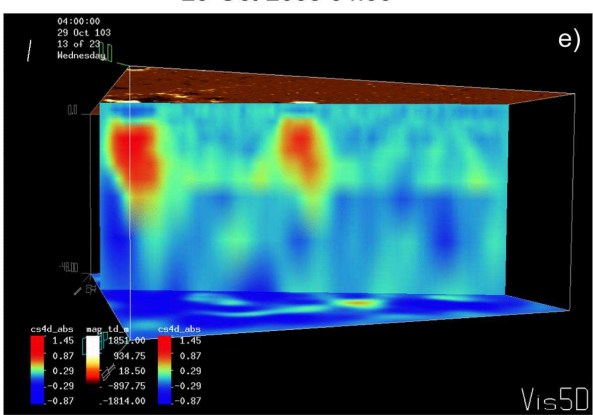

31-Oct-2003 12:00

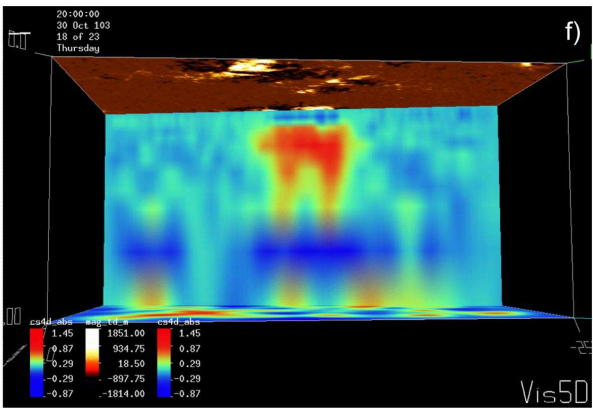

Fig. 10 Subsurface magnetosonic wave-speed structures of the large complex of activity of OctoberNovember 2003, consisting of active regions NOAA 10486 (in the left-hand part of the images), and 10488 (emerging active region in the middle). Red color shows positive wave-speed variations relative to the quiet Sun; the blue color shows the negative variations, which are concentrated near the surface. The upper semitransparent panels show the corresponding MDI magnetograms; the lower panel is a horizontal cut $48 \mathrm{Mm}$ deep. The horizontal size is about $540 \mathrm{Mm}$. The vertical cut goes through both active regions, approximately in the North-South direction crossing the equator, except the image in the right bottom panel, (f), where it goes only through AR 10488 in the East-West direction.

significant time lag. At greater depths the noise level is higher, and it is even more difficult to see the difference in the time evolution relative to the surface magnetic flux.

These results show that the magnetic flux emerges very rapidly from the interior, and that there is no significant (on the scale of few hours) time difference between the evolution of the wave-speed variations associated with the emerging active region and the photospheric magnetic flux. There are indications that the process of the magnetic field concentration, 

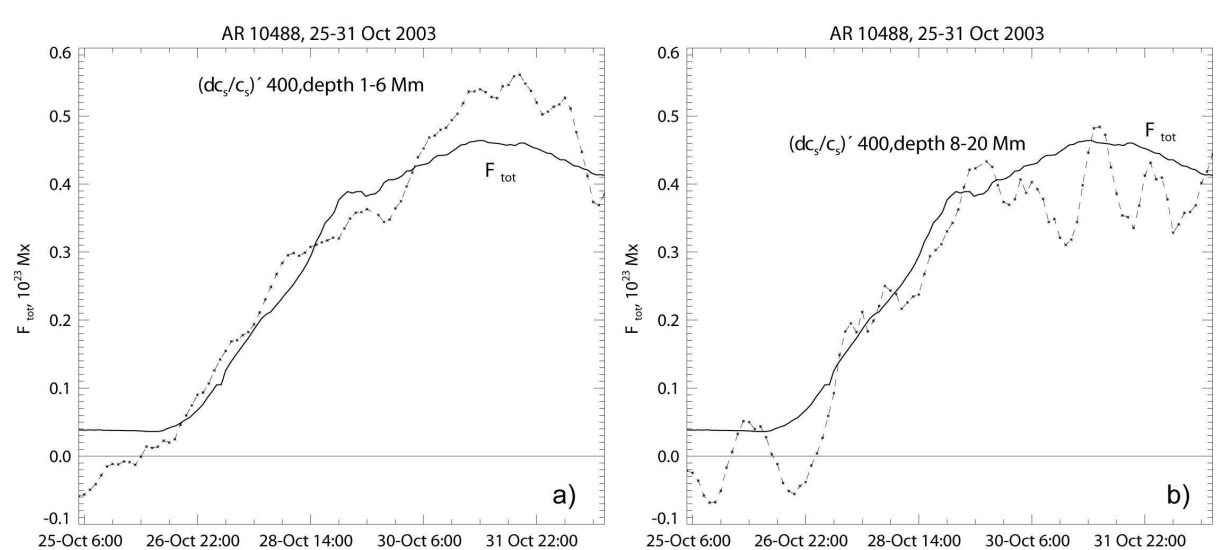

Fig. 11 Evolution of the total unsigned photospheric magnetic flux (solid curve) and the mean relative wavespeed variation (dotted curve with stars) at the depth of 1-6 Mm (left) and 8-20 Mm (right) in the region of the flux emergence of AR 10488.

which forms the active region, first occurs in the subsurface layers, and that then the active region grows because of subsequent flux emergence in this area.

\subsection{Subsurface Flows}

The helioseismology measurements of subsurface flows are obtained from the reciprocal travel times, and generally, are less affected by various kind of uncertainties. They may provide better indicators of the development of active region structures inside the Sun. Figure 12 shows six flow maps at the depth of about $2 \mathrm{Mm}$ for various stages of evolution of the active region, NOAA 10488, before the emergence, during the initial emergence, and during the developed state. The background gray-scale maps show the corresponding magnetograms.

Prior to the emergence, the maps do not show any specific flow pattern that would indicate development of a large magnetic structure below the surface, except, perhaps, a small shearing flow feature, which appeared near the first magnetic field signal in the center of Figure 12 a-b. During the next 8 hours (Fig. 12k), this feature disappears, and a ring-like magnetic field structure is formed. Within this structure the flows are clearly suppressed, and they remain suppressed during further evolution. Also, at the same type a diverging flow pattern starts developing at the boundaries of the magnetic structures. This pattern is consistent with the expectation that the emerging magnetic structure pulls plasma outside. The divergent flow field becomes stronger as the active region grows (Fig. 12 e), but later, it is replaced by a converging flow pattern around the sunspots (Fig. 12f), which was previously observed beneath sunspots (Zhao et al., 2001).

The strength of the divergent flows is obviously related to the development of active regions, and, perhaps, may be even used for predicting their future evolution. The time evolution of the mean horizontal divergence in the two depth intervals and the photospheric flux is shown in Figure 13. It is quite clear that the divergence at the depth 1-6 Mm started to grow before the magnetic flux, reached maximum in the middle of the flux growth phase, and then was replaced by converging flows. At greater depths, 8-20 Mm (Fig. 13 right), the horizontal flow behavior is not very clear, probably because of higher noise, or because 

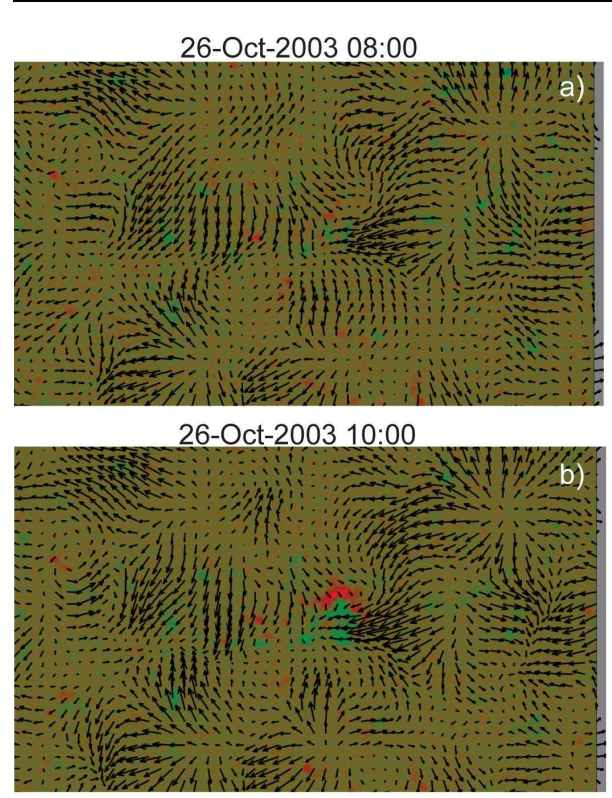

26-Oct-2003 18:00

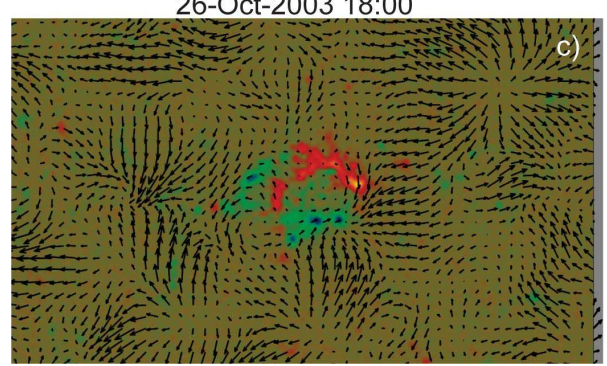

27-Oct-2003 14:00

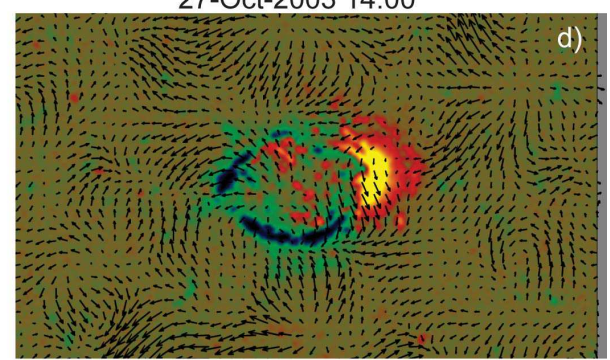

28-Oct-2003 02:00

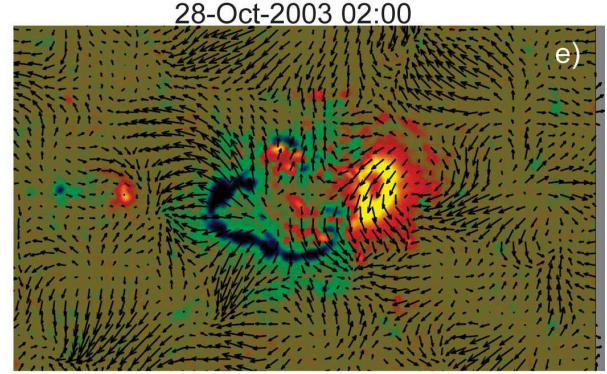

31-Oct-2003 00:00

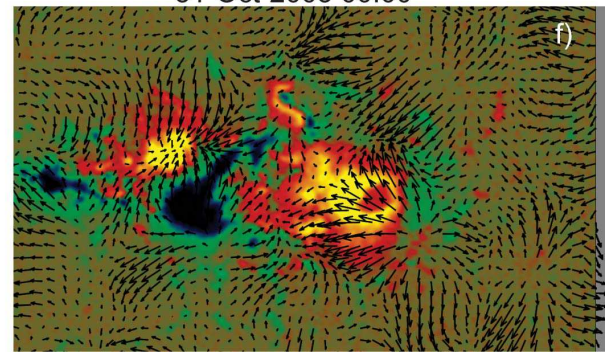

Fig. 12 Evolution of subsurface flows at the depth of $2 \mathrm{Mm}$ below the photosphere during the emergence and growth of AR 10488, on 26-31 October, 2003. The flow maps are obtained by the time-distance technique using 8-hour time series of full-disk Doppler images from SOHO/MDI. The maximum horizontal velocity is approximately $1 \mathrm{~km} / \mathrm{s}$. The background image is the corresponding photospheric magnetogram (red and blue areas show regions of positive and negative polarity of the line-of-sight magnetic field.)

the flow pattern is not as well organized as in the subsurface (6 Mm deep) layer. Perhaps the most significant feature at this depth is the formation of a divergent flow pattern approximately at the time of the formation of convergent flows in the upper subphotospheric layer.

One would expect that during the emergence the plasma is not only pushed outside the magnetic field area but also upward, particularly, in the upper layers. Figure 14 a shows the evolution of the mean vertical flow below the active region at the depth 1-6 Mm. Indeed, upflows dominate at the very beginning of the magnetic flux emergence. However, the signal fluctuates, probably reflecting a complicated structure of the vertical flows. After the emergence phase the vertical flow pattern is dominated by downflows, which are organized around the sunspots.

It seems that the horizontal divergence of subsurface flows is the most sensitive characteristic of the emerging magnetic flux. The divergent flows appear before the initial flux 

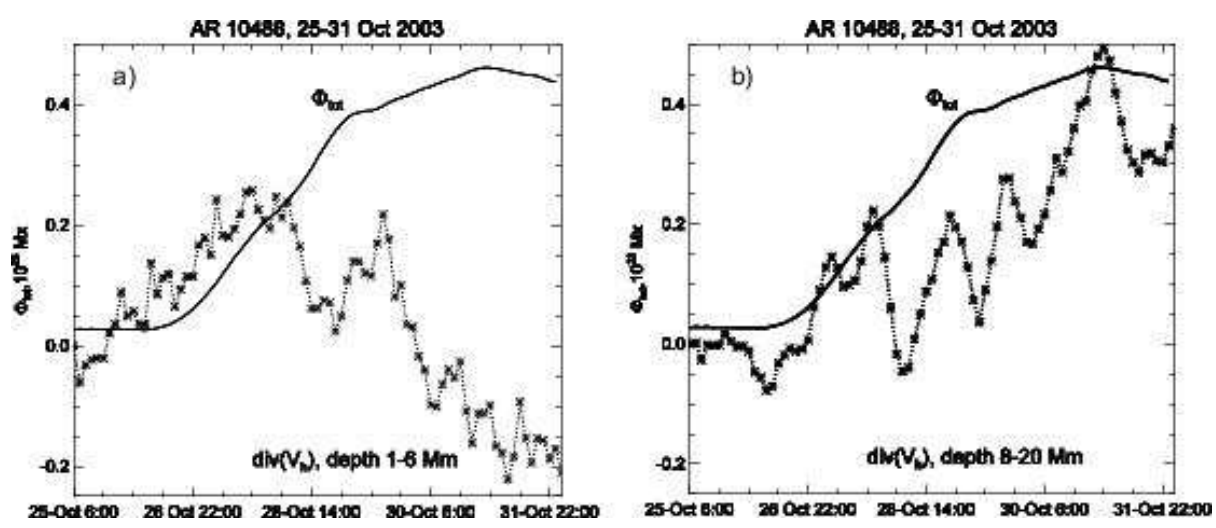

Fig. 13 The evolution of the total unsigned photospheric magnetic flux (solid curve) and the mean divergence of the horizontal flow velocity (dotted curve with stars) at the depth of 1-6 Mm (left) and 8-20 Mm (right) in the region of the flux emergence of AR 10488. The units of $\operatorname{div} V_{h}$ are $3 \cdot 10^{-7} \mathrm{~s}^{-1}$.
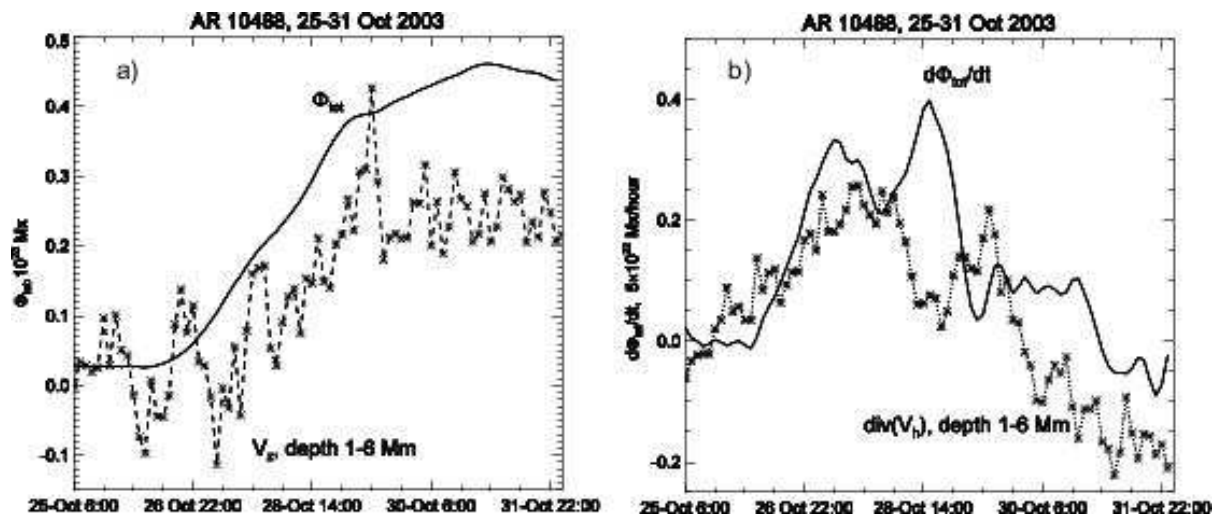

Fig. 14 a) The evolution of the total unsigned photospheric magnetic flux (solid curve) and the mean vertical velocity in $\mathrm{km} / \mathrm{s}$ (dotted curve with stars) at the depth of $1-6 \mathrm{Mm}$ in the region of the flux emergence of AR 10488. The negative velocity corresponds to upflows, and the positive velocity corresponds to downflows. $b$ ) The corresponding changes of the total emerging flux rate and the mean divergence of the horizontal flow components.

emergence, and continue to evolve in correlation with the magnetic flux. Figure 14b shows a comparison of the mean horizontal divergence and the total magnetic rate. Evidently, there were two or three peaks of the magnetic emergence rate. The flow divergence shows two peaks, which are shifted relative to the flux rate. It is unclear whether these peaks precede or follow the magnetic flux emergence events. Obviously, this relationship requires further investigation. Similar flow patterns have been studied by Komm et al. (2008) using data from the GONG network and the ring-diagram method of local helioseismology. The initial results are quite encouraging and show the potential of the helioseismic diagnostics. 
a)
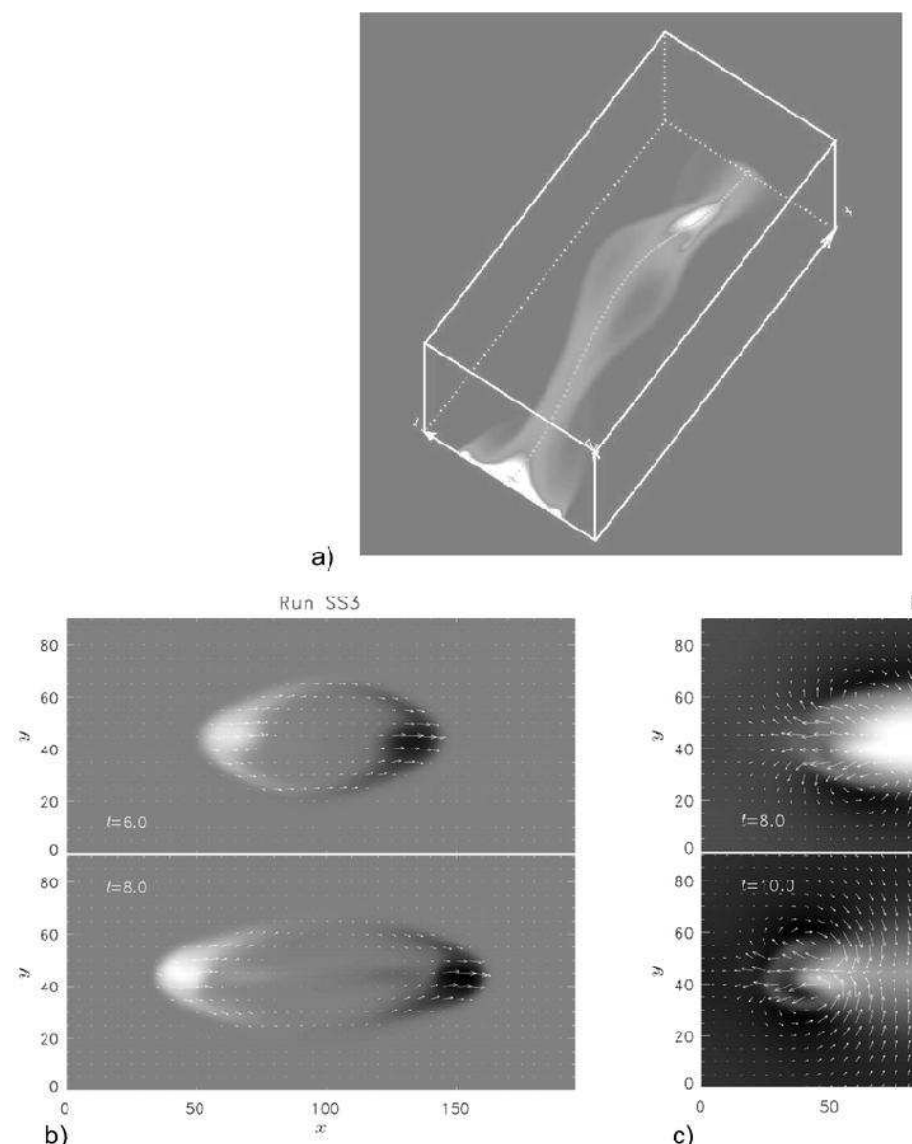

Fig. 15 A theoretical MHD model of emerging magnetic flux tube (Abbett et al. 2000): a) Volume rendering of the magnetic field strength. $b$ ) Vector magnetogram images. The gray-scale background represents the vertical component of the magnetic field (positive values are indicated by the light regions), and the arrows represent the transverse components. $c$ ) Velocity field for the same slice. The gray-scale background represents the vertical component of the velocity (light regions indicate upflows), and the arrows represent the transverse components of the velocity field.

\section{Comparison with Theoretical Models}

The time-distance helioseismology measurements provide new information about the structure and dynamics of emerging active regions. In this paper, we presented in detail the results for a large active region, NOAA 10488, which emerged in October 2003. The results show that the formation of the active region takes about 5 days. During this period the total magnetic flux and the corresponding subsurface wave-speed perturbation grow mostly monotonically. However, the magnetic flux rate reveals two or three peaks of intensive flux emergence; each is about one day long. It appears that the active region is formed by multiple magnetic flux emergence events. The initial magnetic flux emerged very rapidly without any significant perturbation of the Sun's thermodynamic structure or flow field in the place of emergence. There seems to be a short lead in the growth of the subsurface wave speed perturbation relative to the mean magnetic flux, but this relationship is rather uncertain. A 
localized shearing flow seems to be formed few hours before the initial flux emergence, and then disappears soon after the emergence. The active region has a elliptical shape, with the magnetic field concentrated at the boundaries. The plasma flows are suppressed inside this structure. In the outer region, the plasma flows are dominated by divergent flow, driven by the expanding magnetic structures. The flow divergence at the depth of 1-6 Mm shows a few-hour lead relative to the magnetic flux. It grows during the emergence phase until approximately the mid-point of the flux growth curve. After this, the divergence is sharply reduced and then is replaced by predominantly converging flows around sunspots. Approximately, at the same time a divergent flow pattern is formed in the deeper interior (depth 8-20 Mm). The vertical flow pattern is quite complicated. The results do not show strong upflows prior to the emergence, as one might expect. In general, the vertical flow pattern is highly intermittent. There is an evidence of predominant upflows during the initial stage of emergence, but after this the mean flow beneath the active region is directed downwards. It seems that there is an interesting correlation with some time lag between the flow divergence and the flux emergence rate. However, at this stage it is unclear whether the changes in the flow divergence precede or follow the flux rate.

It is interesting that the magnetic structure of this active region, in particular, its elliptical shape is very similar to a model of emerging magnetic flux tube of Abbett et al. (2000) (Fig. 15). The model also predicts a divergent flow pattern similar to the observed one. However, the strong upflow at the beginning of emergence (Fig. 15k, top panel) is not detected in our observations. Thus, the process of emergence and formation of active regions requires further observational and theoretical studies.

\section{Discussion and Future Perspectives}

The recent observations and modeling reveal some interesting features of the properties of emerging magnetic flux and associated dynamics on the solar surface and in the upper convection zone. In particular, the new statistical study of the variations of the tilt angle of bipolar magnetic regions during the flux emergence questions the current paradigm that the magnetic flux emerging on the solar surface represents large-scale magnetic flux ropes ( $\Omega$ loops) rising from the bottom of the convection zone. The flux rope models predict that the tilt angle is a result of the Coriolis effect acting on a plasma flow inside the flux tube, and thus the tilt should depend on latitude, the amount of magnetic flux and relax after the emergence when the Coriolis force vanishes. The observations indeed show the predicted latitudinal dependence (the Joy's law) and indicate that the tilt is formed below the surface. However, there is no evidence of the dependence on the amount of magnetic flux and no evidence for the relaxation of the tilt angle towards the East-West direction. Contrary, the tilt angle tends to relax to the Joy's law value. Perhaps, the Joy's law reflects not the dynamics of the rising flux tubes but the orientation of the toroidal magnetic field lines below the surface as suggested by Babcock (1961).

The observations of the surface flows from SOHO/MDI prior and during the emergence of a large active regions, AR 10488, in October 2003, show strong localized vertical flows just prior the flux emergence and during the initial stage. It is curious that the direction of the flows, namely, an upflow in the area of the leading polarity and a downflow in the following polarity, is consistent with the predictions of the rising flux rope theories. However, observations of some other active regions do not show this (Pevtsov and Lamb, 2006). Also, the data do not show large-scale flow patterns on the surface, which would indicate emergence of a large flux-rope structure. 
The local helioseismology results obtained by both, the time-distance and ring-diagram techniques, show large-scale outflows beneath the surface during most of the emergence phase, and also formation of converging flows around the magnetic structure of sunspots. However, the structure of the vertical flows remains unclear. There is an indication of upflows mixed with downflows at the beginning of emergence, but then the downflows dominate. In the case of AR 10488, there were two or three major flux emergence events. The photospheric magnetic flux rate and subsurface flow divergence show two or three peaks, which are not in phase, but it is unclear if the flux rate precedes the variation of the flow divergence or follows it.

From the observations it is obvious that the multiple flux emergence events over several days plays important role in the formations and maintaining the magnetic structure of the large active region. This reminds the idea of a common 'nest' in the deep interior (Castenmiller et al., 1986). However, such nests have not been found in the helioseismic images of the subphotospheric magnetosonic wave speed variations, which are currently obtained up to the depth of 40-50 Mm. The wave speed images reveal that the emerging magnetic flux structures travel very fast in the upper convection zone, with a speed of at least $1 \mathrm{~km} / \mathrm{s}$. This makes very difficult the detection of these structures before the magnetic field becomes visible on the surface. Thus, it is difficult to use the helioseismology measurements for advanced predictions of emerging active regions. However, it should be possible to use the measurements of both, the wave speed variations and flow velocities, for predicting the growth and and decay of active regions and, perhaps, the complexity of their magnetic structure. This task will require a substantial statistical analysis of emerging active regions by methods of local helioseismology.

Thus, despite the significant new information from helioseismology and magnetography the main questions formulated in Introduction about the origin and physical properties of the emerging magnetic remain unanswered. The recent results from the SOHO spacecraft and GONG network show that for further investigations it is necessary to improve the local helioseismology techniques, extending their coverage into the deep convection zone, carry out statistical studies using uninterrupted solar oscillation data (such as will be available from the Solar Dynamics Observatory mission), and also develop realistic MHD numerical simulations for understanding the physics of magnetic structures in the turbulent convection zone and for supporting the helioseismology observations.

Acknowledgements I wish to thank all the organizers and participants of this workshop, and, in particular, Dr Andre Balogh. This work was supported by the International Space Science Institute (Bern).

\section{References}

W. P. Abbett, G. H. Fisher, and Y. Fan, Astrophys. J. 540, 548 (2000).

H. W. Babcock, Astrophys. J. 133, 572 (1961).

E. E. Benevolenskaya, J. T. Hoeksema, A. G. Kosovichev, and P. H. Scherrer, Astrophys. J. Lett. 517, L163 (1999).

A. C. Birch, A. G. Kosovichev, and T. L. Duvall, Jr., Astrophys. J. 608, 580 (2004).

A. Brandenburg, Astrophys. J. 625, 539 (2005).

P. Caligari, F. Moreno-Insertis, and M. Schussler, Astrophys. J. 441, 886 (1995).

M. J. M. Castenmiller, C. Zwaan, and E. B. J. van der Zalm, Solar Phys. 105, 237 (1986).

S. Couvidat, A. C. Birch, and A. G. Kosovichev, Astrophys. J. 640, 516 (2006). 
S. D'Silva. In: K. L. Harvey (ed.): The Solar Cycle, Vol. 27 of Astronomical Society of the Pacific Conference Series, 168 (1992).

S. D'Silva and R. F. Howard, Solar Phys. 151, 213 (1994).

Y. Fan, G. H. Fisher, and A. N. McClymont, Astrophys. J. 436, 907 (1994).

G. H. Fisher, Y. Fan, and R. F. Howard, Astrophys. J. 438, 463 (1995).

L. Gizon and A. C. Birch, Astrophys. J. 571, 966 (2002).

V. M. Grigor'ev, L. V. Ermakova, and A. I. Khlystova, Astronomy Letters 33, 766 (2007).

G. E. Hale, F. Ellerman, S. B. Nicholson, and A. H. Joy, Astrophys. J. 49, 153 (1919).

J. W. Harvey, F. Hill, R. Hubbard, J. R. Kennedy, J. W. Leibacher, J. A. Pintar, P. A. Gilman, R. W. Noyes, A. M. Title, J. Toomre, R. K. Ulrich, A. Bhatnagar, J. A. Kennewell, W. Marquette, J. Patrón, O. Saá, and E. Yasukawa, Science 272, 1284 (1996).

R. F. Howard, Solar Phys. 167, 95 (1996).

R. F. Howard, Journal of Astrophysics and Astronomy 21, 119 (2000).

R. Howe, J. Christensen-Dalsgaard, F. Hill, R. Komm, J. Schou, M. J. Thompson, and J. Toomre, Advances in Space Research 40, 915 (2007).

J. M. Jensen, T. L. Duvall, Jr., B. H. Jacobsen, and J. Christensen-Dalsgaard, Astrophys. J. Lett. 553, L193 (2001).

R. Komm, S. Morita, R. Howe, and F. Hill, Astrophys. J. 672, 1254 (2008).

A. G. Kosovichev, Astrophys. J. Lett. 469, L61 (1996).

A. G. Kosovichev, T. L. . J. Duvall, and P. H. Scherrer, Solar Phys. 192, 159 (2000).

A. G. Kosovichev and T. L. Duvall, Jr. In: F. P. Pijpers, J. Christensen-Dalsgaard, and C. S. Rosenthal (eds.): SCORe'96 : Solar Convection and Oscillations and their Relationship, Vol. 225 of Astrophysics and Space Science Library, 241 (1997).

A. G. Kosovichev and T. L. Duvall, Jr. In: R. Howe, R. W. Komm, K. S. Balasubramaniam, and G. J. D. Petrie (eds.): Subsurface and Atmospheric Influences on Solar Activity, Vol. 383 of Astronomical Society of the Pacific Conference Series, 59 (2008).

A. G. Kosovichev and J. O. Stenflo, Astrophys. J. Lett. 688, L115 (2008).

E. N. Parker, Astrophys. J. 408, 707 (1993).

E. N. Parker, Astrophys. J. 433, 867 (1994).

A. Pevtsov and J. B. Lamb. In: J. Leibacher, R. F. Stein, and H. Uitenbroek (eds.): Solar MHD Theory and Observations: A High Spatial Resolution Perspective, Vol. 354 of Astronomical Society of the Pacific Conference Series, 249 (2006).

P. H. Scherrer, R. S. Bogart, R. I. Bush, J. T. Hoeksema, A. G. Kosovichev, J. Schou, W. Rosenberg, L. Springer, T. D. Tarbell, A. Title, C. J. Wolfson, I. Zayer, and MDI Engineering Team, Solar Phys. 162, 129 (1995).

H. U. Schmidt. In: K. O. Kiepenheuer (ed.): Structure and Development of Solar Active Regions, Proc. IAU Symposium No. 35, 95 (1968).

M. Schüssler, Astronomische Nachrichten 326, 194 (2005).

L. Tian and Y. Liu, Astron. Astrophys. 407, L13 (2003).

Y.-M. Wang and N. R. Sheeley, Jr., Astrophys. J. 375, 761 (1991).

J. Zhao, A. G. Kosovichev, and T. L. Duvall, Jr., Astrophys. J. 557, 384 (2001).

G. Zhou, J. Wang, Y. Wang, and Y. Zhang, Solar Phys. 244, 13 (2007). 